\title{
Aerosol Delivery Through Adult High Flow Nasal Cannula With Heliox and Oxygen
}

\author{
Patricia A Dailey RRT, Robert Harwood MSA RRT-NPS FAARC, Kyle Walsh, \\ James B Fink PhD RRT FAARC, Tina Thayer RRT, Greg Gagnon, and \\ Arzu Ari PhD RRT PT CPFT FAARC
}

\begin{abstract}
BACKGROUND: Heliox (helium-oxygen mixture) has been shown to reduce turbulence and improve aerosol delivery in a range of clinical settings. We questioned whether heliox as compared with oxygen via high-flow nasal cannula (HFNC) would affect aerosol delivery. We hypothesized that heliox would have a significant effect on aerosol delivery as compared with oxygen with both quiet and distressed breathing patterns. METHODS: A vibrating mesh nebulizer was placed at the inlet of a humidifier via HFNC with small adult cannula distal to the heated-wire circuit with prongs placed into simulated nares with a T-shaped trap and absolute filter connected to a breath simulator set to adult quiet and distressed breathing parameters. Albuterol sulfate $\mathbf{( 0 . 0 8 3 \%}$ $2.5 \mathrm{mg} / 3 \mathrm{~mL}$ ) was aerosolized with heliox (80:20) and oxygen $(100 \%)$ at 10, 30, and $50 \mathrm{~L} / \mathrm{min}$. Drug eluted from the filter was assayed with UV spectrophotometry $(276 \mathrm{~nm})$. Descriptive statistics, Kruskal-Wallis test, and Mann-Whitney $U$ test were used for data analysis. $P<.05$ was considered statistically significant. RESULTS: Increasing flows with heliox and oxygen significantly decreased percentage inhaled dose (inhaled dose) of aerosol with a quiet breathing pattern $(P=.02$ and $P=.030$, respectively). In contrast, with a distressed breathing pattern, inhaled dose at $10 \mathrm{~L} / \mathrm{min}$ was lower than at 30 and $50 \mathrm{~L} / \mathrm{min}(P=.009$ and $P=.01$, respectively) with both oxygen and heliox $(P=.009$ and $P=.009$, respectively). Despite a trend to higher aerosol deposition with heliox versus oxygen, the differences were not significant. CONCLUSIONS: With a distressed breathing pattern, aerosol delivery was greater at 30 and $50 \mathrm{~L} / \mathrm{min}$ than with a quiet breathing pattern. Trends toward higher inhaled dose with heliox during HFNC were not significant. Key words: high-flow nasal cannula; heliox; aerosol; jet nebulizer; vibrating mesh nebulizer. [Respir Care 2017;62(9):1186-1192. (C) 2017 Daedalus Enterprises]
\end{abstract}

\section{Introduction}

Patients with asthma in respiratory distress have an increased work of breathing due to increased airway resis-

\footnotetext{
At the time of this study, Ms Dailey was affiliated with Baystate Medical Center, Springfield, Massachusetts. MrWalsh, Ms Thayer, and Mr Gagnon are affiliated with Baystate Medical Center, Springfield, Massachusetts. Mr Harwood and Dr Fink are affiliated with the Division of Respiratory Therapy, School of Health Professions, Georgia State University, Atlanta, Georgia. Dr Arzu is affiliated with the Department of Respiratory Care, Texas State University, San Marcos, Texas. Dr Fink is affiliated with Aerogen Pharma Corp, San Mateo, California.
}

Ms Dailey is currently a Science Liaison for Aerogen, Ltd. Dr Fink is Chief Science Officer for Aerogen Pharma Corp and discloses relationships with Dance Biopharm, Parion, Bayer, Boerhinger Ingleheim, No- tance caused by inflammation and bronchospasm in the airways. Historically, heliox (helium-oxygen mixture) has been used in the treatment of severe exacerbation of patients with acute obstructive airway disease to improve laminar flow, homogeneous gas distribution, $\mathrm{CO}_{2}$ elimination, and tidal volumes; to reduce transpulmonary pressure requirement; to decrease work of breathing; and to

\footnotetext{
vartis, ONY, Aridis, the World Health Organization, and Ansun. Dr Ari has disclosed a relationship with Bayer Pharmaceuticals. The other authors have disclosed no conflicts of interest.

Correspondence: Patricia A Dailey RRT, 112 Kimberly Drive, Manchester, CT 06040. E-mail: PDailey@aerogen.com.
}

DOI: $10.4187 /$ respcare. 05127 
improve aerosol delivery. ${ }^{1}$ Studies on the effect of heliox in acute severe asthma have demonstrated rapid improvement in air-flow obstruction, dyspnea, and ventilation during administration, suggesting that heliox therapy could be a valuable therapeutic bridge to conventional treatment. ${ }^{2,3}$ Heliox therapy is concentration-dependent and is traditionally administered with high-flow oxygen delivery systems, such as a valved mask with a reservoir and a nonrebreather mask for spontaneously breathing patients or through a mechanical ventilator for intubated patients. The advent of high-flow nasal cannula (HFNC) has provided the clinician with another option for delivery of heliox therapy.

Asthma exacerbation with acute respiratory failure frequently requires escalation of care, such as invasive ventilation, noninvasive ventilation (NIV), and more recently the use of HFNC. Clinicians understand that invasive procedures such as intubation pose many risks, and the use of noninvasive respiratory support is preferred. Although NIV is less invasive than intubation, research has identified several drawbacks to the use of NIV in patients with acute respiratory distress. Asthma patients in respiratory distress are often reluctant to have a confining mask over their face, which adds to an already claustrophobic feeling. Intolerance and discomfort to NIV can lead to early interruption in up to $22 \%$ of patients. ${ }^{4} \mathrm{HFNC}$ application is a respiratory support mode better tolerated by patients that has been shown to have an equivalent benefit for patients in respiratory distress. ${ }^{5}$

Heated HFNC therapy provides flows up to $50-60 \mathrm{~L} / \mathrm{min}$ of water-saturated gas ( $\left.44 \mathrm{mg} \mathrm{H}_{2} \mathrm{O} / \mathrm{L}\right)$ at body temperature $\left(37^{\circ} \mathrm{C}\right)$. Most systems utilize an air/oxygen blender, which allows for a $\mathrm{F}_{\mathrm{IO}_{2}}$ range of 0.21-1.00. HFNC has been described as a useful respiratory support modality in the stabilization of patients in respiratory distress. ${ }^{6,7}$ Recently, the use of HFNC has become popular as a first-line support modality for patients in respiratory distress, due to ease of use and improved patient tolerance compared with NIV. ${ }^{7}$ Poor tolerance of NIV has been reported as a reason for intubation in $5-25 \%$ of the cases in hypoxemic patients..$^{8,9}$

Patients requiring respiratory support often require aerosolized medications, including $\beta$ agonists. Aerosol delivery through HFNC has been described in the literature in infant, pediatric, and adult in vitro models. Réminiac ${ }^{10}$ reported reductions in inhaled dose with increased flows and that simulated respiratory distress as compared with quiet breathing did not hamper aerosol delivery. Bhashyam et $\mathrm{al}^{11}$ demonstrated that aerosols could be efficiently delivered through HFNC at flows of $3 \mathrm{~L} / \mathrm{min}$, with inhaled dose up to $28 \%$ using adult-size cannulas.

Heliox has been described as a method to improve response to bronchodilator aerosols in children and adults, providing more laminar flow through constricted airways

\section{QUICK LOOK}

\section{Current knowledge}

Aerosol delivery through high-flow nasal cannula (HFNC) has been described in the literature in infant, pediatric, and adult in vitro models. Aerosol delivery with heliox via HFNC in the pediatric model tested at 3 and $6 \mathrm{~L} / \mathrm{min}$ has been shown to increase aerosol delivery 2 -fold at the higher flow as compared with oxygen. With quiet breathing, as flow increases, the percentage of dose inhaled decreases.

\section{What this paper contributes to our knowledge}

Our study showed that aerosols can be efficiently delivered through HFNC. We confirmed previous reports that flow is inversely related to aerosol delivery with quiet breathing. We also demonstrated that heliox aerosol delivery trended higher at the higher flows tested ( 30 and $50 \mathrm{~L} / \mathrm{min}$ ) but not at the lower flow $(10 \mathrm{~L} / \mathrm{min}$ ). In contrast, the simulated respiratory distress pattern resulted in a significant increase in aerosol delivery at mid and high flows, with higher flows associated with a greater percentage of inhaled dose. Using heliox as the carrier gas with a distressed breathing pattern improved delivery of aerosol by $3-4 \%$ at all flows tested. Further studies are required to indicate whether this difference is meaningful in distressed patients.

and reducing impactive losses of aerosol in transit from large to smaller airways. ${ }^{1}$ Ari et $a l^{5}$ compared heliox versus oxygen as a carrier gas via HFNC in a pediatric in vitro model with pediatric cannula and circuit between 2 and $6 \mathrm{~L} / \mathrm{min}$, with heliox improving aerosol delivery at the high flow.

We were curious about the effects of flow and heliox on aerosol delivery with an adult HFNC and circuit application simulating adult parameters with both quiet and distressed breathing patterns. The goal of this study was to evaluate the potential for delivering aerosol via heated HFNC utilizing an adult model in an in vitro study with a range of HFNC flows used in clinical application and toe compare the impact of heliox on aerosol delivery via HFNC.

\section{Methods}

We used an HFNC system (Optiflow and MR 850 humidifier, Fisher \& Paykel, Auckland, New Zealand) including an autofill chamber, a heated-wire breathing circuit, and a small adult-size Optiflow nasal cannula. The system is thermostatically controlled and autoregulates to body temperature $\left(37^{\circ} \mathrm{C}\right)$ and $100 \%$ relative humidity set 
in the invasive mode, allowing the delivery (per label) of fully saturated gas $\left(44 \mathrm{mg} / \mathrm{L}\right.$ at $\left.37^{\circ} \mathrm{C}\right)$ up to $60 \mathrm{~L} / \mathrm{min}$.

Operating gas applied to the inlet of the humidifier for oxygen was administered with a MaxVenturi (MaxTech, Salt Lake City, Utah) flow meter, and heliox (80:20) was administered via cylinder with a heliox regulator (Western Medica, Portland, Oregon) and oxygen flow meter (Amvex, Richmond Hill, Ontario, Canada) through a T-piece connected to the inlet (dry side) of the humidifier chamber with a vibrating mesh nebulizer (Solo, Aerogen, Galway, Ireland). The nasal cannula was placed distal to the heatedwire circuit into an adapter with loose orifices simulating nares with a $\mathrm{T}$-shaped trap positioned above the filter to collect non-aerosolized liquid and a collecting filter (Respirgard 303, CareFusion, San Diego, California) attached to a breath simulator (Dual Phase Control Small Animal Respiratory Model 55, Harvard Apparatus, Holliston, Mas-

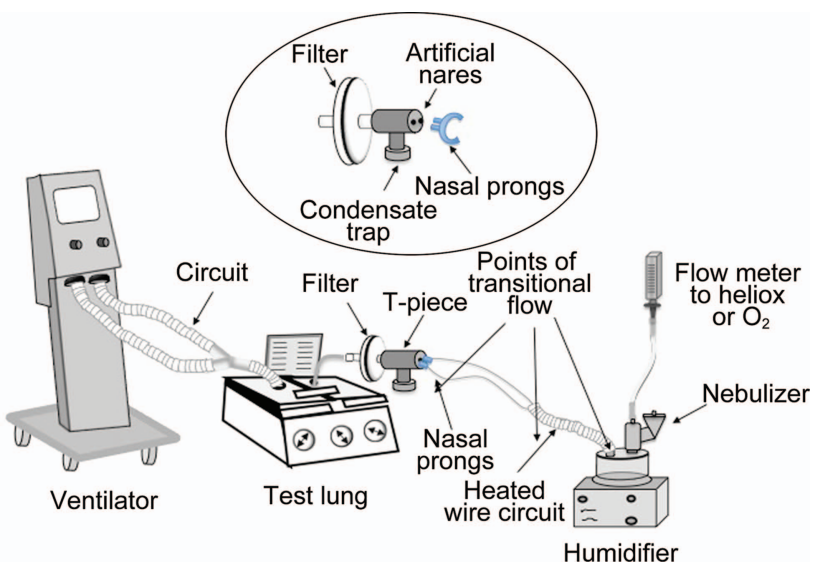

Fig. 1. Experimental setup of the study. Gas from heliox or $\mathrm{O}_{2}$ cylinders passes through a flow meter attached to a T-piece with a nebulizer at the inlet of the humidifier to a heated-wire circuit and nasal prongs to a T-piece and filter attached to one side of the test lung, with a rigid bar attached to other test lung compartment attached to the ventilator. Ventilation of the test lung compartment moves the other compartment, simulating a spontaneous breathing pattern. The inset shows a disassembled view of the interface of the nasal prong, T-piece with artificial nares, condensate trap, and collecting filter. Three primary points of transitional flow occur while gas passes through the humidifier into the circuit, from the circuit to the nasal cannula, and from the nasal prongs into the artificial nares. From Reference 5, with permission. sachusetts). The filter assembly was placed above the trap to collect aerosol and to prevent contamination from rainout (Fig. 1).

This study was based on the methodology previously reported with a pediatric HFNC. ${ }^{5}$ Differences with the adult application include: greater range of flows, a larger bore circuit and cannula, and use of ventilatory pattern representative of adults with quiet and distressed breathing. The flows of 10,30 , and $50 \mathrm{~L} / \mathrm{min}$ with oxygen and heliox 80:20 were based on a range of flows used in clinical practice. A VT Plus HF gas flow analyzer (Fluke Biomedical, Everett, Washington) was calibrated according to the manufacturer's recommendations and was used to confirm settings on the breath simulator as well as oxygen and heliox flow before each run. This setup was used for all experiments (Fig. 2).

\section{Experiment 1: Quiet Breathing Pattern}

The breathing simulator was set to a quiet adult breathing pattern: tidal volume of $500 \mathrm{~mL}$, breathing frequency of 16 breaths/min, and inspiratory-expiratory ratio of 1:2. Albuterol sulfate $(2.5 \mathrm{mg} / 0.5 \mathrm{~mL})$ was placed in the vibrating mesh nebulizer and nebulized to completion $(n=3)$.

\section{Experiment 2: Distressed Breathing Pattern}

The breathing simulator was set to simulate a respiratory distressed breathing pattern: tidal volume of $750 \mathrm{~mL}$, breathing frequency of 30 breaths/min, and inspiratoryexpiratory ratio of $1: 1$. Albuterol sulfate $(5 \mathrm{mg} / 1.0 \mathrm{~mL})$ was nebulized to completion $(n=5)$. We increased the dose of albuterol with distressed breathing, to be more representative of the clinical dose used for those conditions and to ensure that the assay was within the limits of quantification at higher flows, consequently increasing inhaled drug mass (mg) while having little impact on inhaled dose efficiency (\% inhaled dose).

Albuterol was eluted from each filter with $10 \mathrm{~mL}$ of $0.01 \mathrm{~N}$ hydrochloric acid, with agitation for $3 \mathrm{~min}$ and assayed with UV spectrophotometry $(276 \mathrm{~nm})$. The amount of albuterol eluted from each filter was reported as inhaled

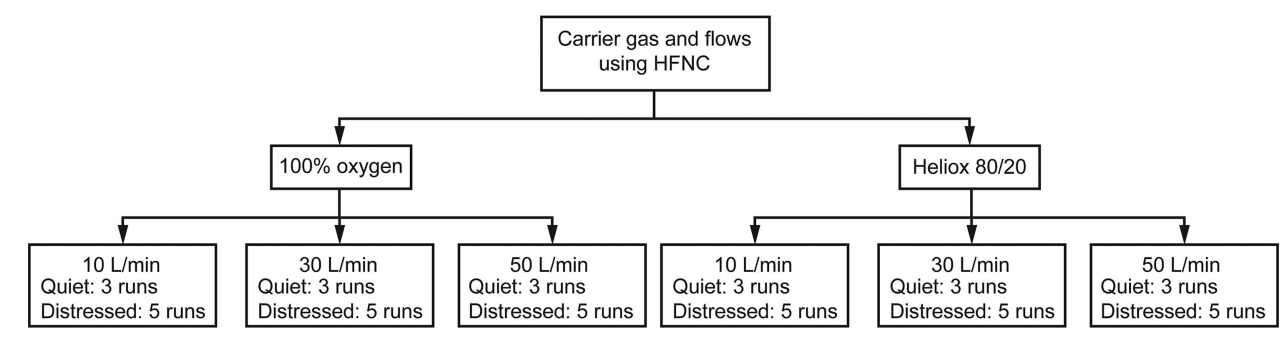

Fig. 2. Flow chart detailing the number of runs in quiet and distressed breathing patterns. HFNC $=$ high-flow nasal cannula. 
Table 1. Inhaled Mass and Inhaled Dose Collected on the Filter After Nebulization With Vibrating Mesh Through an Adult High-Flow Nasal Cannula With Quiet Adult Simulated Breathing Pattern

\begin{tabular}{|c|c|c|c|c|c|c|c|}
\hline \multirow{2}{*}{ Gas } & \multicolumn{2}{|c|}{ 10-L/min Flow } & \multicolumn{2}{|c|}{ 30-L/min Flow } & \multicolumn{2}{|c|}{ 50-L/min Flow } & \multirow[b]{2}{*}{$P$} \\
\hline & $\begin{array}{c}\text { Inhaled Mass (mg) } \\
\quad(n=3)\end{array}$ & $\begin{array}{l}\text { Inhaled Dose }(\%) \\
\quad(n=3)\end{array}$ & $\begin{array}{l}\text { Inhaled Mass (mg) } \\
\quad(n=3)\end{array}$ & $\begin{array}{c}\text { Inhaled Dose }(\%) \\
\quad(n=3)\end{array}$ & $\begin{array}{l}\text { Inhaled Mass (mg) } \\
\qquad(n=3)\end{array}$ & $\begin{array}{c}\text { Inhaled Dose }(\%) \\
\quad(n=3)\end{array}$ & \\
\hline Oxygen $(100 \%)$ & $0.667 \pm 0.032$ & $26.7 \pm 1.29$ & $0.289 \pm 0.029$ & $11.6 \pm 1.17$ & $0.088 \pm 0.004$ & $3.5 \pm 0.17$ & .002 \\
\hline Heliox $(80: 20)$ & $0.684 \pm 0.059$ & $27.4 \pm 2.37$ & $0.356 \pm 0.022$ & $14.2 \pm 0.89$ & $0.394 \pm 0.468$ & $5.88 \pm 1.73$ & .003 \\
\hline$P$ & & .70 & & .10 & & .11 & \\
\hline
\end{tabular}

Table 2. Results of Inhaled Mass and Inhaled Dose Collected on the Filter After Nebulization With Vibrating Mesh Through an Adult High-Flow Nasal Cannula With a Distressed Adult Simulated Breathing Pattern

\begin{tabular}{|c|c|c|c|c|c|c|c|}
\hline \multirow[b]{2}{*}{ Gas } & \multicolumn{2}{|c|}{ 10-L/min Flow } & \multicolumn{2}{|c|}{ 30-L/min Flow } & \multicolumn{2}{|c|}{ 50-L/min Flow } & \multirow[b]{2}{*}{$P$} \\
\hline & $\begin{array}{l}\text { Inhaled Mass (mg) } \\
\quad(n=5)\end{array}$ & $\begin{array}{c}\text { Inhaled Dose }(\%) \\
\quad(n=5)\end{array}$ & $\begin{array}{l}\text { Inhaled Mass (mg) } \\
\quad(n=5)\end{array}$ & $\begin{array}{l}\text { Inhaled Dose }(\%) \\
\quad(n=5)\end{array}$ & $\begin{array}{l}\text { Inhaled Mass (mg) } \\
\qquad(n=5)\end{array}$ & $\begin{array}{c}\text { Inhaled Dose }(\%) \\
\quad(n=5)\end{array}$ & \\
\hline Oxygen (100\%) & $0.652 \pm 0.164$ & $13 \pm 3$ & $1.644 \pm 0.241$ & $33 \pm 5$ & $1.263 \pm 0.085$ & $25 \pm 2$ & .004 \\
\hline Heliox (80:20) & $0.873 \pm 0.159$ & $17 \pm 3$ & $1.757 \pm 0.241$ & $35 \pm 5$ & $1.501 \pm 0.203$ & $30 \pm 4$ & .005 \\
\hline$P$ & & .056 & & .55 & & .056 & \\
\hline
\end{tabular}

5-mg nominal dose. Tidal volume $750 \mathrm{~mL}$, breathing frequency 30 breaths/min, and inspiratory-expiratory ratio 1:1 with oxygen and heliox 80:20 at 10, 30, and 50 L/min.

mass (mg) and percentage of nominal dose (\% inhaled dose) delivered distal to the nares during each experiment.

\section{Statistical Analysis}

Descriptive statistics were used to calculate the mean and SD for each gas type, flow, and breathing pattern tested in this study. The Kruskal-Wallis test was used to determine significant differences among 10, 30, and $50 \mathrm{~L} / \mathrm{min}$ using heliox and oxygen at quiet and distressed conditions. The Mann-Whitney U test was used to compare aerosol delivery with heliox and oxygen at each flow tested in this study. Differences between quiet and distressed breathing patterns were determined with the Mann-Whitney U test with $P<.05$ considered statistically significant.

\section{Results}

\section{Aerosol Delivery With Quiet Breathing Pattern}

Mean inhaled dose of drug delivered distal to the nares via HFNC ranged from 3.5 to $26.70 \%$ for $100 \%$ oxygen and from 5.9 to $27.40 \%$ for $80: 20$ heliox (Table 1). Increasing flows with heliox and oxygen significantly decreased inhaled dose of aerosol in this simulated adult lung model with a quiet breathing pattern $(P=.02$ and $P=.030$, respectively). Inhaled dose with heliox at $10 \mathrm{~L} / \mathrm{min}$ was greater than that at $30 \mathrm{~L} / \mathrm{min}(P=.005)$ and $50 \mathrm{~L} / \mathrm{min}$ $(P=.001)$, whereas aerosol deposition at $50 \mathrm{~L} / \mathrm{min}$ was lower than at $30 \mathrm{~L} / \mathrm{min}(P=.030)$. All comparisons between flows in aerosol delivery with oxygen were statistically significant $(P<.05)$. Although aerosol deposition trended higher with heliox than oxygen, the differences found between these gases at 10,30, and $50 \mathrm{~L} / \mathrm{min}$ were not significant $(P=.70, P=.10$, and $P=.11$, respectively). There was a greater difference in inhaled dose between the 2 gases at 30 and $50 \mathrm{~L} / \mathrm{min}$ as compared with $10 \mathrm{~L} / \mathrm{min}$.

\section{Aerosol Delivery With Distressed Breathing Pattern}

In contrast to quiet breathing, delivery efficiency with distressed breathing was greater, ranging from 13 to $33 \%$ for oxygen and from 17 to $35 \%$ for heliox (Table 2). The percentages of inhaled dose delivered with both oxygen and heliox at different flows were statistically significant ( $P=.004$ and $P=.005$, respectively). Aerosol delivery with oxygen at $10 \mathrm{~L} / \mathrm{min}$ was significantly lower than that at 30 and $50 \mathrm{~L} / \mathrm{min}(P=.009$ and $P=.01$, respectively $)$. Although no statistical difference was found between 30 and $50 \mathrm{~L} / \mathrm{min}$ using oxygen $(P=.056)$, delivery efficiency of HFNC with oxygen at $30 \mathrm{~L} / \mathrm{min}$ was greater than at $50 \mathrm{~L} / \mathrm{min}$ and may be considered clinically important. Aerosol deposition with heliox at $10 \mathrm{~L} / \mathrm{min}$ was significantly lower than at 30 and $50 \mathrm{~L} / \mathrm{min}(P=.009$ and $P=.009$, 
respectively). No statistical difference was found in aerosol drug delivery between 30 and $50 \mathrm{~L} / \mathrm{min}$ using heliox $(P=.09)$. Regardless of the type of gas used in this study, aerosol deposition was greater at $30 \mathrm{~L} / \mathrm{min}$ in this simulated adult distressed breathing pattern. Comparisons of oxygen and heliox inhaled dose showed no significant difference at 10,30 , or $50 \mathrm{~L} / \mathrm{min}(P=.056, P=.55$, and $P=.056$, respectively).

\section{Comparisons of Quiet and Distressed Breathing Pattern in Aerosol Delivery With Oxygen and Heliox}

Aerosol delivery efficiency via HFNC with both oxygen and heliox at $10 \mathrm{~L} / \mathrm{min}$ with distressed breathing was less than with the quiet breathing pattern $(P=.036)$ but greater at 30 and $50 \mathrm{~L} / \mathrm{min}(P=.036)$. Comparisons of quiet and respiratory distressed breathing patterns in aerosol delivery with heliox showed the same trend. Whereas inhaled dose with heliox at $10 \mathrm{~L} / \mathrm{min}$ was statistically lower in a distress condition than in a quiet breathing pattern, increasing flows to 30 or $50 \mathrm{~L} / \mathrm{min}$ significantly improved delivery efficiency of HFNC in this simulated adult distressed breathing ( $P=.036$ and $P=.030$, respectively).

\section{Discussion}

This is the first reported model of inhaled aerosol in an adult model comparing heliox and oxygen during HFNC, comparing drug delivery with 3 commonly used flows (10, 30 , and $50 \mathrm{~L} / \mathrm{min}$ using a quiet and distressed breathing pattern. With the quiet breathing pattern, inhaled dose was inversely related to flow, and differences between oxygen and heliox trended higher as flow increased. At $10 \mathrm{~L} / \mathrm{min}$, heliox had no additional effect on aerosol delivery compared with oxygen. The inhaled dose of $26.70-27.74 \%$ reported at $10 \mathrm{~L} / \mathrm{min}$ with oxygen during quiet breathing was similar to that reported by Bhashyam et $\mathrm{al}^{11}$ using a similar adult nasal cannula, despite the lower $3 \mathrm{~L} / \mathrm{min}$ used. Our findings of decreased aerosol delivery with increases in flow with quiet breathing were consistent with those of Ari et al, ${ }^{5}$ Perry et al, ${ }^{12}$ and Réminiac et al. ${ }^{10}$

Ari et $a^{5}$ reported that inhaled aerosol was inversely proportional to flow with both heliox and oxygen administered via high-flow nasal cannula in a pediatric model. Aerosol delivery with oxygen at 3 and $6 \mathrm{~L} / \mathrm{min}$ was 10.65 and $1.95 \%$, and with heliox, it was 11.41 and $5.42 \%$, respectively. Although heliox did not make a significant difference at the lower flow, it was significant $(P=.01)$ at the higher flow compared with oxygen, producing a 2-fold increase in aerosol delivery.

Perry et $\mathrm{a}^{12}$ also reported a negative correlation between flows and inhaled dose across infant, pediatric, and adult breathing parameters. In their model, a vibrating mesh aerosol generator was placed with a proprietary adapter between the source of humidified gas and the adult cannula, simulating flow ranges administered to infants, children, and adults, resulting in $0.20-2.50 \%$ nominal dose with most of the drug $(62-80 \%)$ collected in the adapter. Although increasing the cannula size increased aerosol delivery, they concluded that the amount of drug delivered at the majority of flows and cannula sizes was not sufficient to produce a clinical response. In contrast, our results revealed much higher drug delivery, 3.5-35.0\% inhaled dose, which could be considered sufficient to support clinical efficacy.

Réminiac et $\mathrm{al}^{10}$ evaluated inhaled dose from a highflow nasal cannula with flows of 30,45 , and $60 \mathrm{~L} / \mathrm{min}$ with $60 \%$ oxygen at $31^{\circ} \mathrm{C}$, using a cast adult upper airway. They reported an inhaled dose of 3.0-10.2\% with lower aerosol delivery with increasing flows with both quiet and distressed ventilatory patterns. Differences in magnitude of their reported inhaled dose (3.0-10.5\%) versus our findings (3.5-33.0\%) with oxygen are probably due to the model used, with their upper-airway cast reducing the aerosol dose en route to the trachea of the model, compared with our measurements at the nares.

Réminiac et $\mathrm{al}^{10}$ reported greater aerosol dose across all flows compared with simulated quiet breathing. They reasoned that the higher mean inspiratory flow of $45 \mathrm{~L} / \mathrm{min}$ with distressed breathing allowed a greater proportion of gas containing aerosol to be inhaled compared with the $15-\mathrm{L} / \mathrm{min}$ mean inspiratory flow with quiet breathing. In contrast, at $10 \mathrm{~L} / \mathrm{min}$, we observed a decrease in inhaled dose with the higher inspiratory flow of distressed breathing.

The premise of high-flow oxygen administration is to meet and exceed the patient's inspiratory flow demand, reducing the amount of ambient gas that is entrained. As inspiratory flow exceeds the rate of gas administered, more room air is inhaled, reducing $\mathrm{F}_{\mathrm{IO}_{2}}$. When aerosol is continuously added and mixed in the administered gas, the better the inspiratory flow matches the delivered gas, the greater proportion of aerosol is inhaled. As the operating gas flow exceeds inspiratory flow, a lower proportion of aerosol-containing gas is inhaled. Consequently, during distressed breathing, Réminiac et $\mathrm{al}^{10}$ observed greater inhaled dose with flows of $30-60 \mathrm{~L} / \mathrm{min}$. This was consistent with our findings at 30 and $50 \mathrm{~L} / \mathrm{min}$.

We evaluated 80:20 heliox because this is the highest concentration that can be safely used in clinical settings. Because the effects of heliox are concentration-dependent on decreasing transitional and turbulent flow, 80:20 heliox would provide the maximum impact on aerosol delivery during HFNC. Although there was a trend toward greater deposition with 80:20 heliox, it failed to meet statistical significance and may not be clinically relevant. Consequently, the lower concentrations of heliox that could be 
used in patients requiring higher $\mathrm{F}_{\mathrm{IO}_{2}}$ would be expected to provide even less benefit.

We learned from our previous study with the pediatric HFNC model that as total flow through the HFNC increases, the inhaled dose decreases with both gases, and a smaller proportion of the emitted aerosol is inhaled. ${ }^{5}$ The vibrating mesh nebulizer produces consistent output of aerosol/min over time in both oxygen and heliox. ${ }^{13} \mathrm{We}$ also found a difference in aerosol delivery with heliox versus oxygen with mid and high flows. We questioned whether the lower density of heliox as compared with oxygen had the potential to reduce turbulent flow and improve aerosol delivery. ${ }^{7,14-17}$ Our results indicated that at the higher flows tested with a simulated quiet breathing pattern heliox did improve aerosol delivery at $30 \mathrm{~L} / \mathrm{min}$ (22.40\% increase in aerosol delivery) and $50 \mathrm{~L} / \mathrm{min}(68.00 \%$ increase in aerosol delivery). However, impact on aerosol delivery at the lower flow of $10 \mathrm{~L} / \mathrm{min}$ was only $2.60 \%$. At the lower flow of $10 \mathrm{~L} / \mathrm{min}$ and the, inspiratory flow of the model at $30 \mathrm{~L} / \mathrm{min}$, the mixing of room air in with the heliox, probably caused reduction of the concentration below that threshold at which heliox provided a mechanical advantage. As flow came closer to and exceeded the inspiratory flow of the model, a higher concentration of heliox was inhaled, increasing the chance of a reduction in transitional or turbulent flows at the interface of the cannula and the airway. It was likely that a combination of these elements resulted in the greater differences in inhaled dose for $\mathrm{O}_{2}$ and heliox as flow increased.

Reduction of inhaled dose with increasing flows with quiet breathing could be explained by one of 2 mechanisms: (1) increased turbulence and impactive loss of aerosol with increasing delivery flows and (2) the dilution of aerosol within the stream of gas flowing to the cannula. Heliox is known to reduce turbulent and transitional flow patterns compared with air or oxygen, which has been associated with reduced impactive losses of aerosol. In our hands, the use of heliox resulted in small improvements in aerosol delivery with our model, increasing the inhaled dose by up to $68.00 \%$ compared with oxygen at $50 \mathrm{~L} / \mathrm{min}$ with quiet pattern.

As gas flow exceeded the inspiratory flow of the model, a greater proportion of gas containing aerosol bypassed the airway and was released to atmosphere. This is supported by reports of Réminiac et al, ${ }^{10}$ who compared a quiet and distressed breathing pattern with higher inspiratory flows, reporting an almost 2 -fold greater inhaled dose than at the quiet settings they used. This suggests that increased inspiratory flows had a greater impact on improvement of inhaled dose during administration of higher gas flows than heliox. This observation has clinical relevance, since most patients requiring HFNC are likely to have some level of respiratory distress with higher-than-quiet inspiratory flows. Consequently, the use of quiet adult parameters probably underestimates the inhaled dose for patients with distressed breathing patterns.

\section{Clinical Implications}

This is the first study to show the effect of increasing flow on aerosol delivery in the adult quiet and distressed breathing model with HFNC and similarly the first to show the impact of heliox on aerosol delivery at this range of flows. Optimal aerosol delivery via HFNC can be achieved through various mechanisms, all of which should be based on matching the therapy parameters to the individual patient. The better the HFNC flow meets the patient inspiratory flow demand, the higher the inhaled dose. Aerosol delivery with heliox through HFNC trended to improve inhaled dose at mid and high flows as compared with oxygen but failed to be statistically significant in our model. This begs the question of whether those trends would be clinically meaningful in patients in respiratory distress with severe airway obstruction. Heliox adds cost to therapy and may be cost-prohibitive. For this reason, the application of heliox with HFNC and aerosol delivery should be considered if heliox makes a clinical difference in terms of work of breathing or distribution of ventilation and/or if the patient is already receiving heliox. Otherwise, utilizing flows required to meet the patient's inspiratory flow demand and oxygen requirements and to reduce work of breathing during aerosol therapy will improve aerosol delivery. Removing the patient from high flow is not a viable option because it interrupts oxygen delivery and respiratory support.

\section{Limitations}

This model did not take into account exhaled gas or use an anatomically correct model of the nares and upper airway. The breathing patterns were limited to one set of parameters for quiet and distressed breathing and did not differentiate opened versus closed mouth breathing. ${ }^{10} \mathrm{We}$ evaluated aerosol delivery with only a small adult cannula, and based on the report from Perry et al, we might expect aerosol delivery to be improved with the use of larger-size cannulas. ${ }^{12}$ Future studies to determine the impact of cannula size are needed. It would be interesting to see results for a 60:40 mixture of heliox, but lower concentrations are likely to result in even lower differences in the percentage of dose inhaled both with our model and with patients.

This was an in vitro study, and we do not know how heliox may affect the patient airway and the potential impact on aerosol delivery in an obstructed patient airway. In addition, scintigraphy and clinical response studies would be useful to further evaluate the role of aerosol administration with heliox via HFNC. 


\section{Conclusions}

Aerosol can be efficiently delivered through an adult HFNC, and is a viable application for aerosol delivery. Inhaled dose of aerosol varied with increases in flows and breathing patterns for both oxygen and heliox. Although inhaled dose trended higher with heliox than with oxygen, the use of heliox as a carrier gas did not significantly improve aerosol delivery at the flows tested. Aerosol delivery with simulated respiratory distressed breathing patterns increased aerosol delivery as compared with quiet breathing patterns.

The ability to use aerosols in patients receiving HFNC with or without heliox provides the clinician with options for patient care. To optimize aerosol delivery, clinical application of aerosol with the HFNC should be done using flows that meet patient inspiratory demands during distressed breathing. Clinical studies evaluating clinical response and outcomes should be done to determine efficacy and safety.

\section{REFERENCES}

1. Hess DR, Fink JB, Venkataraman ST, Kim IK, Myers TR, Tano BD. The history and physics of heliox. Respir Care 2006;51(6):608-612.

2. Kass JE, Terrigino C. The effect of heliox in acute severe asthma: a randomized controlled trial. Chest 1999;116(2):296-300.

3. Kass JE Castriotta RJ. Heliox therapy in acute severe asthma. Chest 1995;107(3):757-760.

4. Messika J, Ben Ahmed K, Gaurdry S, Miguel-Montanes R, Rafat C, Sztrymf B, et al. Use of high-flow nasal cannula oxygen therapy in subjects with ARDS: a 1-year observational study. Respir Care 2015; 60(2):162-169.

5. Ari A, Harwood R, Sheard M, Dailey P, Fink JB. In vitro comparison of heliox and oxygen in aerosol delivery using pediatric high flow nasal cannula. Pediatr Pulmonol 2011;46(8):795-801.

6. Lenglet H, Sztrymf B, Leroy C, Brun P, Dreyfuss D, Ricard JD. Humidified high flow nasal oxygen during respiratory failure in the emergency department: feasibility and efficacy. Respir Care 2012; 57(11):1873-1878

7. Spivey S, Ashe T, Dennis R, Graham R, Melton B, Croft S, et al. Assessment of high flow nasal cannula therapy use in the emergency department setting: observations of practice across four systems. Respir Ther 2015;10(1):30-34.

8. Gotera C, Díaz Lobato S, Pinto T, Winck JC. Clinical evidence on high flow oxygen therapy and active humidification in adults. Rev Port Pneumol 2013;19(5):217-227.

9. Frat JP, Brugiere B, Ragot S, Chatellier D, Veinstein A, Goudet V, et al. Sequential application of oxygen therapy via high-flow nasal cannula and noninvasive ventilation in acute respiratory failure: an observational pilot study. Respir Care 2015;60(2):170-178.

10. Réminiac F, Vecellio L, Heuzé-Vourc'h N, Petitcollin A, Respaud R, Cabrera M, et al. Aerosol therapy in adults receiving high flow nasal cannula oxygen therapy. J Aerosol Med Pulm Drug Deliv 2016; 29(2):134-141.

11. Bhashyam AR, Wolf MT, Marcinkowski AL, Saville A, Thomas K, Carcillo JA, Corcoran TE. Aerosol delivery through nasal cannulas: an in vitro study. J Aerosol Med Pulm Drug Deliv 2008;21(2):181188.

12. Perry SA, Kesser KC, Geller DE, Selhorst DM, Rendle JK, Hertzog $\mathrm{JH}$. Influences of cannula size and flow rate on aerosol drug delivery through the Vapotherm humidified high-flow nasal cannula system. Pediatr Crit Care Med 2013;14(5):e250-e256.

13. O'Callaghan C, White J, Jackson J, Crosby D, Dougill B, Bland H. The effects of heliox on the output and particle-size distribution of salbutamol using jet and vibrating mesh nebulizers. J Aerosol Med 2007;20(4):434-444.

14. Papamoschou D. Theoretical validation of the respiratory benefits of helium-oxygen mixtures. Respir Physiol 1995;99(1):183-190.

15. Kim IK, Saville AL, Sikes KL, Corcoran TE. Heliox-driven albuterol nebulization for asthma exacerbations: an overview. Respir Care 2006;51(6):613-618.

16. Corcoran TE, Gamard S. Development of aerosol drug delivery with helium oxygen gas mixtures. J Aerosol Med 2004;17(4):299-309.

17. Goode ML, Fink JB, Dhand R, Tobin MJ. Improvement in aerosol delivery with helium-oxygen mixtures during mechanical ventilation. Am J Respir Crit Care Med 2001;163(1):109-114. 